
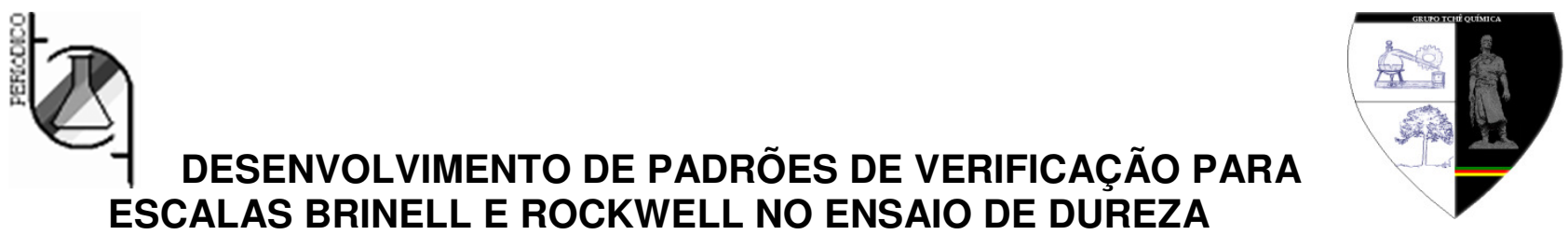

\title{
DEVELOPMENT OF VERIFICATION STANDARDS FOR BRINELL AND ROCKWELL HARDNESS TESTS
}

\author{
FABRICIO, Daniel Antonio Kapper ${ }^{1 *}$; TREVISAN, Lisiane ${ }^{2}$; SARTORI, Cecília ${ }^{1}$ \\ 1 Instituto Federal de Santa Catarina, Campus Chapecó. \\ Av. Nereu Ramos, 3450-D, CEP 89813-000, Chapecó - SC, Brasil \\ (fone: +55 493313 1245) \\ ${ }^{2}$ Instituto Federal de Educação, Ciência e Tecnologia do Rio Grande do Sul - IFRS, Campus Farroupilha \\ Av. São Vicente 785, CEP 95180-000, Farroupilha - RS, Brasil \\ (fone: +55 543260 2400) \\ ${ }^{*}$ Autor correspondente \\ e-mail: danielkapper@gmail.com
}

Received 162 November 2018; received in revised form 28 November 2018; accepted 29 November 2018

\section{RESUMO}

O ensaio de dureza é um dos ensaios mecânicos destrutivos mais utilizados pela indústria metalmecânica. A garantia dos resultados medidos deve-se principalmente ao fator humano, que está envolvido nas etapas de verificação, ajuste e operação dos equipamentos de medição. Sendo assim, o objetivo principal deste artigo é projetar e construir padrões de verificação para as escalas Brinell (HB) e Rockwell (HRC) a partir de aços carbono baixa liga, submetidos a diferentes tipos de tratamento térmico. Para garantir a confiabilidade dos valores de dureza dos padrões de verificação, os mesmos serão submetidos a medições em equipamentos calibrados por laboratórios pertencentes à Rede Brasileira de Calibração (RBC). Os resultados mostraram valores semelhantes com base em dez medições realizadas sobre a superfície do padrão.

Palavras-chave: Ensaio Mecânico; Dureza; Metrologia; Padrões de Verificação.

\section{ABSTRACT}

The hardness test is one of the most important mechanical tests used by the metal-mechanical industry. The quality assurance of the measured results is mainly due to the human factor, which is involved in the verification, adjustment, and operation of the measuring equipment. Therefore, the main goal of this paper is to design and develop verification standards for Brinell (HB) and Rockwell (HRC) scales from low alloy carbon steels subjected to different types of heat treatment. In order to assure the reliability of the hardness values of the verification standards, they have been measured on equipment calibrated by laboratories belonging to the Brazilian Calibration Network (RBC). The results showed similar values based on ten measurements made on the standards surface.

Keywords: Mechanical Testing; Hardness; Measurement; Verification Standards. 


\section{INTRODUÇÃO}

O ensaio de dureza é utilizado na indústria para 0 controle de qualidade em diversos processos de fabricação metalúrgicos. Este controle através do ensaio de dureza pode ser feito na inspeção de materiais no recebimento ou durante as etapas de fabricação de um componente (GARCIA et al., 2000).

O ensaio de dureza pode ser considerado como a medida da resistência de um material à deformação plástica (CALLISTER, 2012). Vários fatores alteram os valores de dureza medidos: composição química do material, microestrutura, existência (ou não) de camada superficial, entre outros; além de parâmetros voltados ao próprio ensaio, tais como: etapa de preparação da superfície, paralelismo, aplicação da carga e leitura da endentação realizada na superfície do material (SOUZA, 2000; ABNT, 2008).

O objetivo principal deste trabalho é desenvolver 4 padrões de verificação para a escala HRC (Rockwell C) e HB (Brinell) para o uso na verificação dos valores de dureza pelos durômetros existentes no Instituto Federal do Rio Grande do Sul - Campus Farroupilha.

Para que se possa construir o padrão de verificação, será necessário em primeiro lugar escolher o material metálico a partir do qual serão construídos os padrões. Partindo do material metálico, será necessária a realização da etapa de usinagem e tratamento térmico de cada um dos materiais, etapa muitas vezes limitadas pelos equipamentos disponíveis.

O uso de padrões de calibração e de verificação podem ser confundidos facilmente. Os padrões de calibração servem para que possam ser feitos ajustes no equipamento para que a leitura do durômetro se aproxime do valor descrito no certificado do padrão de calibração (THOLEN, 2011; GUM, 2012). Já os padrões de verificação são usados diariamente para a verificação dos valores medidos pelo durômetro. Portanto, os padrões de verificação não servem para ajustar o equipamento (SILVA NETO, 2012).

\section{MATERIAL E MÉTODOS}

Este trabalho pretende desenvolver uma pesquisa quantitativa com uma abordagem experimental, onde se determina um objeto de estudo, selecionam-se as variáveis que seriam capazes de influenciá-lo, definem-se as formas de controle e de observação dos efeitos que a variável produz no objeto.

Como há uma grande combinação entre valores de dureza, escala e materiais metálicos que podem ser utilizados no ensaio de dureza, optou-se pela escolha dos padrões de verificação com base nos materiais metálicos disponíveis para a realização deste trabalho.

As escalas HRC (Rockwell C) e HB (Brinell) foram escolhidas em função das escalas trabalhadas em sala de aula com os alunos dos cursos técnicos e de graduação do Instituto Federal do Rio Grande do Sul. Assim, este trabalho está relacionado à interação pesquisaensino, mostrando assim a importância do conhecimento de metrologia nas disciplinas de graduação do curso superior em engenharia mecânica. Através do uso dos padrões de verificação, os alunos podem verificar o funcionamento e estimar o erro de medição existente em uma medição realizada em um componente metálico qualquer.

Para a realização da parte experimental foi realizada a confecção de corpos de prova de dureza em aço carbono SAE 1020 e em um aço ferramenta de médio teor de carbono DIN 1.2367. A composição química do material metálico usado está de acordo com descrito pela norma ABNT NBR NM 87 (ABNT, 2000).

Estes aços foram escolhidos devido à grande possibilidade de combinação entre dureza e tratamento térmico que permite uma ampla gama de valores de dureza dentro da mesma escala. As dimensões escolhidas para a construção dos padrões estão apresentadas na Figura 1.

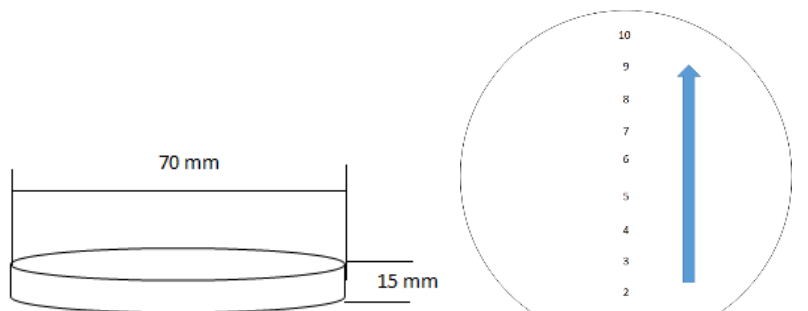

Figura 1. Dimensões do padrão de verificação e direção das medidas realizadas.

As dimensões foram escolhidas em função do material disponível, seguindo a recomendação da norma ISO 6508-3 (ISO, 2015), que especifica que os blocos de verificação para o ensaio de dureza devem ter espessura entre 6 e $16 \mathrm{~mm}$, sendo que a espessura mínima aceita para aço é 
de $12 \mathrm{~mm}$. Além disso, os blocos de verificação devem ser isentos de magnetismo.

Foram confeccionados corpos de prova nos materiais descritos anteriormente, os quais foram usinados e fresados em máquina fresadora universal. Posteriormente ao processo de fresa, o material foi usinado em uma retífica com pastilha de diamante para melhor acabamento superficial.

O valor de rugosidade superficial não se altera nos valores de dureza dependendo do nível de força aplicado. A etapa de polimento é suficiente para escala Rockwell $C$ e para escala com uso de indentador esférico se a força aplicada for menor que $100 \mathrm{kgf}$ (LOW, 2001).

Após o processo de usinagem, os corpos de prova foram submetidos aos tratamentos térmicos de normalização, têmpera e revenimento:

- Para o material SAE 1020 foi realizado o processo de normalização com aquecimento até $900^{\circ} \mathrm{C}$ e resfriamento lento ao ar.

- Para o aço ferramenta médio carbono o mesmo foi temperado em forno a vácuo em $1020^{\circ} \mathrm{C}$ e resfriado em nitrogênio com uma pressão de 3 bar.

- Após o aquecimento e resfriamento, a amostra em aço ferramenta DIN 1.2367 foi revenida em duas temperaturas, de $600^{\circ} \mathrm{C}$ e $300^{\circ} \mathrm{C}$, atingindo as durezas de 38 e $55 \mathrm{HRC}$, respectivamente.

Em função da variabilidade do processo de fabricação dos padrões por tratamento térmico (composição química, tamanho de grão, temperaturas de trabalho, atmosfera, equipamentos de medição de dureza), é possível observar diferenças entre os valores reais e de projeto de dureza para os padrões fabricados.

A incerteza de medição dos valores de dureza foi calculada segundo o Guia para a Expressão da Incerteza de Medição (JCGM, 2008), utilizando como apoio o Guia da Rede Metrológica do Rio Grande do Sul (RMRS, 2013).

\section{RESULTADOS E DISCUSSÃO}

Os resultados de dureza após a etapa de tratamento térmico para os materiais podem ser visualizados na Tabela 1.
Tabela 1. Valores projetados de dureza em função da etapa de tratamento térmico.

\begin{tabular}{c|c|c}
\hline Material & $\begin{array}{c}\text { Tratamento } \\
\text { térmico }\end{array}$ & $\begin{array}{c}\text { Intervalo } \\
\text { projetado }\end{array}$ \\
\hline SAE & Recozido & $180-200 \mathrm{HB}$ \\
1020 & & \\
SAE & Normalização & $260-280 \mathrm{HB}$ \\
1020 & Têmpera e & $51-52 \mathrm{HRC}$ \\
DIN & revenimento & revenido $300^{\circ} \mathrm{C}$ \\
1.2367 & Têmpera e & $36-39 \mathrm{HRC}$ \\
DIN & rêmido $650^{\circ} \mathrm{C}$ \\
1.2367 & revenimento & reveniden
\end{tabular}

O aço SAE 1020 apresentou valores entre 95 e 110 HRB após o tratamento de normalização, o que indica uma dureza maior relativa ao mesmo material quando comparado ao estado recozido.

Os resultados para o aço DIN 1.2367 apresentaram faixas distintas de dureza após o revenimento em função das diferentes temperaturas de revenimento adotadas experimentalmente. O objetivo dessa variação é criar duas faixas de dureza distintas para o mesmo material e poder qualificar duas faixas de dureza Rockwell com o mesmo aço tratado termicamente.

Os resultados do perfil de dureza aplicado nos corpos de prova para cada material mostraram uma média e incerteza conforme os resultados da Tabela 2 para os padrões da escala Rockwell C e, a Tabela 3 para os padrões da escala Brinell.

As medidas foram realizadas em um durômetro calibrado de um laboratório universitário acreditado segundo a ABNT NBR ISO/IEC 17025 (ABNT, 2017).

Tabela 2. Valores de dureza obtidos experimentalmente para escala Rockwell C.

\begin{tabular}{c|c|c}
\hline Medida & $\begin{array}{c}\text { Padrão 35 } \\
\text { HRC }\end{array}$ & $\begin{array}{c}\text { Padrão 56 } \\
\text { HRC }\end{array}$ \\
\hline 1 & 33,2 & 44,9 \\
2 & 34,5 & 53,2 \\
3 & 35,8 & 53,5 \\
4 & 35,6 & 53,5 \\
5 & 35,3 & 53,0 \\
6 & 35,5 & 53,3 \\
7 & 35,2 & 53,6 \\
8 & 35,7 & 54,1 \\
9 & 35,2 & 53,5 \\
10 & 35,8 & 54,2 \\
Média & 35,18 & 52,69 \\
Incerteza & 0,83 & 2,89 \\
\hline
\end{tabular}


Tabela 3. Valores de dureza obtidos experimentalmente para escala Brinell.

\begin{tabular}{c|c|c}
\hline Medida & $\begin{array}{c}\text { Padrão 200 } \\
\text { HB }\end{array}$ & $\begin{array}{c}\text { Padrão } \mathbf{2 8 0} \\
\text { HB }\end{array}$ \\
\hline 1 & 197 & 286 \\
2 & 197 & 236 \\
3 & 198 & 274 \\
4 & 199 & 283 \\
5 & 199 & 242 \\
6 & 196 & 276 \\
7 & 198 & 251 \\
8 & 198 & 290 \\
9 & 200 & 287 \\
10 & 198 & 288 \\
Média & 198,00 & 271,30 \\
Incerteza & 1,05 & 18,59 \\
\hline
\end{tabular}

Para a escala HRC, as medições foram realizadas em durômetro com esfera de $2,5 \mathrm{~mm}$ e carga de 187,5 kgf, com uso de um padrão primário. $\mathrm{O}$ durômetro foi submetido a medidas com padrão primário de 46,3 HRC, estando dentro do valor aceitável pelo sistema de qualidade do laboratório escolhido.

Já para a escala Brinell, foi utilizado um durômetro semi-automático com esfera de $2,5 \mathrm{~mm}$ de diâmetro e carga de 187,5 kgf por 15 segundos. A calibração do durômetro foi realizada com o uso de um padrão com 207 HB e incerteza expandida de 4,5 HB a um nível de confiança de $95 \%$.

É importante ressaltar que os corpos de prova foram submetidos ao tratamento térmico sob o mesmo equipamento (forno com aquecimento e atmosfera controlável) e também sob a mesma corrida (mesmo lote) oriundo do processo siderúrgico. Assim, minimizam-se fontes de incerteza oriundas do tratamento térmico (variações de temperatura entre ciclos de tratamento). Além disso, são minimizadas variações de composição química entre corpos de prova.

Em relação aos resultados apresentados na Tabela 2, o valor do desvio padrão do padrão de $280 \mathrm{HB}$ representa $7,55 \%$ do valor médio, enquanto para o padrão de $200 \mathrm{HB}$, o desviopadrão representa $0,58 \%$ do valor médio. A dispersão entre os resultados experimentais representa a maior fonte de incerteza de medição no ensaio de dureza, os valores de desvio padrão estão ligados aos valores de incerteza de medição.
A incerteza de medição foi determinada a partir das seguintes fontes: desvio padrão experimental, com distribuição de probabilidade normal; incerteza herdada do certificado de calibração, também com distribuição de probabilidade normal; e a resolução do identador, com distribuição de probabilidade retangular. A incerteza combinada foi expandida para uma probabilidade de abrangência de $95 \%$.

O valor de uniformidade $\left(\cup_{\text {rel }}\right)$ aceito pela norma ISO 6506-1:2017 (ISO, 2017a) mostra que o valor deve seguir norma ISO 6506-2:2017 (ISO, 2017b) para as realizações das medidas de dureza, com temperatura entre $(23 \pm 5)^{\circ} \mathrm{C}$.

A uniformidade é determinada em função da repetitividade, e esta deve estar dentro do valor determinado em função do intervalo de dureza, conforme descrito na Tabela 4. Os valores descritos na Tabela 4 são valores de uniformidade para padrões primários construídos para serem utilizados ensaios de dureza. Seus valores aceitáveis dependem da escala de dureza que está sendo analisada.

Tabela 4. Valores de uniformidade dos padrões calculados a partir dos resultados obtidos no ensaio de dureza HRC.

\begin{tabular}{c|c|c|c|c}
\hline Uniformidade & $\begin{array}{c}\mathbf{3 5} \\
\text { HRC }\end{array}$ & $\begin{array}{c}\mathbf{5 6} \\
\text { HRC }\end{array}$ & $\begin{array}{c}\mathbf{2 0 0} \\
\text { HB }\end{array}$ & $\begin{array}{c}\mathbf{2 8 0} \\
\text { HB }\end{array}$ \\
\hline$U_{\text {rel }}(\%)$ calculado & 7,4 & 17,7 & 2,02 & 19,90 \\
\hline $\begin{array}{c}U_{\text {rel }}(\%) \text { máximo } \\
\text { aceito pela norma }\end{array}$ & $\begin{array}{c}1,0 \text { a } 0,4 \\
\text { HRC }\end{array}$ & 2,5 & 2,0 \\
\hline
\end{tabular}

O teor de uniformidade encontrado pelos valores medidos depende da dispersão dos resultados e também da qualidade superficial do padrão. A rugosidade superficial do padrão não foi considerada neste projeto. No entanto, conforme descrito em EURAMET (2007), a rugosidade superficial é fundamental para que seja possível obter resultados com reprodutibilidade aceitável.

Os valores demonstrados na Tabela 3 mostram que os padrões construídos por esta metodologia não são aceitáveis para ser utilizados como padrões primários, o que não impede que sejam utilizados como padrões de verificação, objetivo deste trabalho.

Observa-se que o padrão de $200 \mathrm{HB}$ encontra-se mais próximo ao padrão primário. $\mathrm{O}$ material metálico utilizado para este padrão foi apenas recozido, permitindo assim que a microestrutura formada seja mais uniforme, reduzindo assim a dispersão entre os resultados. 


\section{CONCLUSÕES}

Este trabalho descreveu a metodologia usada na construção de padrões de verificação para escalas de dureza Brinnel e Rockwell para duas faixas de valores. Os padrões construídos partiram de dois materiais metálicos amplamente utilizados pela indústria metalúrgica.

Esta metodologia se mostrou adequada para a elaboração de padrões de verificação, mas não para a construção de padrões primários de dureza. Esta conclusão foi possível através dos valores de uniformidade calculados para os valores medidos, sendo que os mesmos se encontram acima dos valores aceitáveis pela norma ISO 6506-2:2017 (ISO, 2017).

Para indicar possível fontes de dispersão dos resultados obtidos, alguns fatores podem ser indicados, tais como a existência de inclusões e de elementos de liga residuais. Estes fatores relacionados à composição química podem acarretar na heterogeneidade da microestrutura (ISO, 2006).

Em relação à dispersão de dados, ainda que tenham sido realizadas 10 medições para cada padrão de verificação construído, não foi realizada a construção de diferentes padrões sob as mesmas condições indicadas. Seriam necessários no mínimo três corpos de prova sob as mesmas condições para garantir a reprodutibilidade da metodologia criada para a confecção de padrões de verificação para estas escalas (JCGM, 2012).

\section{AGRADECIMENTOS}

Ao Conselho Nacional de Desenvolvimento Científico e Tecnológico (CNPq) e ao Instituto Federal do Rio Grande do Sul (IFRS), pelo fomento, conforme Edital PROPPI № 012/2016 - PIBIC-EM/IFRS/CNPq.

À Aços Favorit, empresa parceira neste projeto de pesquisa desenvolvido em conjunto com o $\mathrm{CNPq}$, pelo fornecimento do material utilizado neste trabalho.

À Tramontina Cutelaria S.A. pelo auxílio na realização da etapa de tratamento térmico.

\section{REFERÊNCIAS}

1. ABNT NM ISO 6508-1:2008. Metallic materials - Rockwell hardness test Part 1: Test method (scales A, B, C, D, E, F, G, H, K, N, T). 2008.

2. ASSOCIAÇÃO BRASILEIRA DE NORMAS TÉCNICAS (ABNT). ABNT NBR NM 87:2000. Aço carbono e ligados para construção mecânica - Designação e composição química. 2000.

3. ASSOCIAÇÃO BRASILEIRA DE NORMAS TÉCNICAS (ABNT). NBR ISO/IEC 17025: requisitos gerais para a competência de laboratórios de ensaio e calibração. Rio de Janeiro: ABNT, 2017.

4. CALLISTER, W. D. Ciência e engenharia dos materiais: Uma introdução. 7 ed. LTC: Rio de Janeiro, 2012.

5. EURAMET. Guia para a estimativa da incerteza em medições de dureza. EURAMET/cg-16/v.01, Julho 2007.

6. EURAMET. Guia para estimativa da incerteza de medição em ensaios de dureza - DIMEC/gc-04/v.00. Tradução do documento EURAMET/CG-16/v.01. Guia de Calibração. Setembro de 2008.

7. Garcia, A.; Spim, J.; Santos, C. A. Ensaios dos materiais. 2 ed., LTC: Rio de Janeiro, 2012.

8. ISO 6508-3. Metallic materials: Rockwell hardness test. Part 3: Calibration of reference blocks. ISO, 2015.

9. ISO 6506-1:2017. Metallic materials -Brinell hardness test -- Part 1: Test method. 2017a.

10. ISO 6506-2:2017. Metallic materials -Brinell hardness test -- Part 2: Verification and calibration of testing machines. 2017b.

11. ISO Guide 35:2006. Reference materials General and statistical principles for certification. 2006.

12. JOINT COMMITTEE FOR GUIDES IN METROLOGY. Evaluation of measurement data: guide to the expression of uncertainty in measurement (GUM). JCGM 100, 1. ed. Geneva: BIPM, 2008.

13. JOINT COMMITTEE FOR GUIDES IN METROLOGY. International vocabulary of metrology (VIM): basic and general concepts and associated terms. JCGM 200, 3. ed. Geneva: BIPM, 2012.

14. LOW, S. R. NIST Recommended Practice Guide: Rockwell Hardness of metallic materials. National Institute of Standards and Technology: 2001.

15. RMRS. RM 68 - Incerteza de medição: guia prático do avaliador de laboratórios. 
Revisão 05 . Porto Alegre: Rede

Metrológica RS, 2013.

16. SILVA NETO, J. C. Metrologia e controle dimensional: Conceitos, normas e aplicações. Editora Campus: 2012.

17. SOUZA, S. A. Ensaios mecânicos de materiais metálicos. 5 ed. Editora Blucher: 2000.

18. THOLEN, D. Metrology in service of society: the role of proficiency testing. Accreditation and Quality Assurance. Berlin, v. 16, p. 603-605, 2011.

OPEN ACCESS. This article is licensed under a Creative Commons Attribution 4.0 (CC BY 4.0) International License, which permits use, sharing, adaptation, distribution, and reproduction in any medium or format, as long as you give appropriate credit to the original author(s) and the source, provide a link to the Creative Commons license, and indicate if changes were made. The images or other third-party material in this article are included regulation or exceeds the permitted use, you will need to obtain permission directly from the copyright holder. To view a copy of this license, visit http://creativecommons.org/licenses/by/4.0/. 